\title{
New Insights into the Growth Mechanism and Surface Structure of Palladium Nanocrystals
}

\author{
Byungkwon Lim ${ }^{1, \dagger}$, Hirokazu Kobayashi ${ }^{1, \dagger}$, Pedro H. C. Camargo ${ }^{1}$, Lawrence F. Allard ${ }^{2}$, Jingyue Liu ${ }^{3}(\bowtie)$, and \\ Younan $\mathrm{Xia}^{1}(\triangle)$ \\ ${ }^{1}$ Department of Biomedical Engineering, Washington University, St. Louis, Missouri 63130, USA \\ ${ }^{2}$ Materials Science and Technology Division, Oak Ridge National Laboratory, Oak Ridge, Tennessee 37831, USA \\ ${ }^{3}$ Center for Nanoscience and Department of Chemistry and Biochemistry, University of Missouri-St. Louis, St. Louis, Missouri 63121, USA \\ Received: 24 November 2009 / Revised: 26 December 2009 / Accepted: 20 January 2010 \\ C The Author(s) 2010. This article is published with open access at Springerlink.com
}

\begin{abstract}
This paper presents a systematic study of the growth mechanism for Pd nanobars synthesized by reducing $\mathrm{Na}_{2} \mathrm{PdCl}_{4}$ with $L$-ascorbic acid in an aqueous solution in the presence of bromide ions as a capping agent. Transmission electron microscopy (TEM) and high-resolution TEM analyses revealed that the growth at early stages of the synthesis was dominated by particle coalescence, followed by shape focusing via recrystallization and further growth via atomic addition. We also investigated the detailed surface structure of the nanobars using aberration-corrected scanning TEM and found that the exposed $\{100\}$ surfaces contained several types of defects such as an adatom island, a vacancy pit, and atomic steps. Upon thermal annealing, the nanobars evolved into a more thermodynamically favored shape with enhanced truncation at the corners.
\end{abstract}

\section{KEYWORDS}

Palladium, nanocrystals, growth, coalescence, surface evolution

Metal nanocrystals have attracted growing attention due to their fascinating properties and invaluable applications in catalysis, photonics, sensing, and imaging [1-3]. The physicochemical properties of a metal nanocrystal are determined by a set of parameters such as shape, size, and composition. In particular, shape control of a metal nanocrystal can provide a versatile avenue for tailoring its catalytic activity and selectivity because shape determines the arrangements of atoms on the surface [4-8]. For example, it has been shown that $\mathrm{Pd}$ nanocubes bounded by $\{100\}$ facets can provide a four-fold improvement in specific activity for the formic acid oxidation reaction as compared to $\mathrm{Pd}$ octahedra bounded by $\{111\}$ facets [9]. An exquisite shape control of metal nanocrystals is therefore essential for the maximization of their activity and thus their performance in many catalytic and electrocatalytic applications.

In order to achieve a high-level control over the shape of metal nanocrystals prepared in a solution phase, a fundamental understanding of nucleation and growth mechanisms is a prerequisite. In the classical models, nanocrystals have been considered to grow by atomic addition [10-12]. However, recent experimental and theoretical studies have shown that particle coalescence

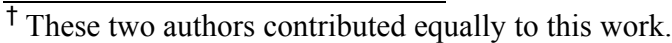

Address correspondence to Younan Xia, xia@biomed.wustl.edu; Jingyue Liu, liuj@umsl.edu 
can also play an important role in the growth process [13-17]. For instance, oriented attachment was proposed as a growth mechanism to account for the formation of aggregates, nanorods, or nanowires for various metals and metal oxides [18-23]. Using in situ transmission electron microscopy (TEM), Alivisatos and co-workers recently provided direct evidence for the involvement of particle coalescence in the growth of Pt nanocrystals [24]. Despite these studies, however, a detailed description of the growth process is still missing, especially for nanocrystals with a specific shape.

Here we present a mechanistic study of the growth of Pd nanobars. Similar to a nanocube, a nanobar is characterized by its six main faces bounded by $\{100\}$ facets except that its dimension is elongated along one direction. As we have demonstrated in a recent study [25], these Pd nanostructures can be easily synthesized in an aqueous solution by reducing $\mathrm{Na}_{2} \mathrm{PdCl}_{4}$ with $L$-ascorbic acid in the presence of bromide ions as a capping agent to promote the formation of $\{100\}$ facets, and the aspect ratio can be controlled by adjusting the reaction temperature. In this paper, we provide new insights into the growth mechanism, with features such as growth via particle coalescence and shape focusing via recrystallization, which have not been considered in previous studies. We have also investigated the detailed surface structure of the Pd nanobars by characterizing them with sub-Ångström resolution, high-angle annular dark-field scanning TEM (HAADF-STEM) and found that the as-synthesized nanobars contained several types of defects such as adatom islands and steps on their exposed $\{100\}$ surfaces.

Figure 1(a) shows a typical TEM image of the as-synthesized $\mathrm{Pd}$ nanobars obtained at $80^{\circ} \mathrm{C}$. The particles were all bar-shaped and had relatively low aspect ratios. The average (avg.) aspect ratio, width, and length of the nanobars were $1.18,8.4 \mathrm{~nm}$, and $9.8 \mathrm{~nm}$, respectively (Fig. 1(b); see also Figs. S-1(a) and S-1(b)

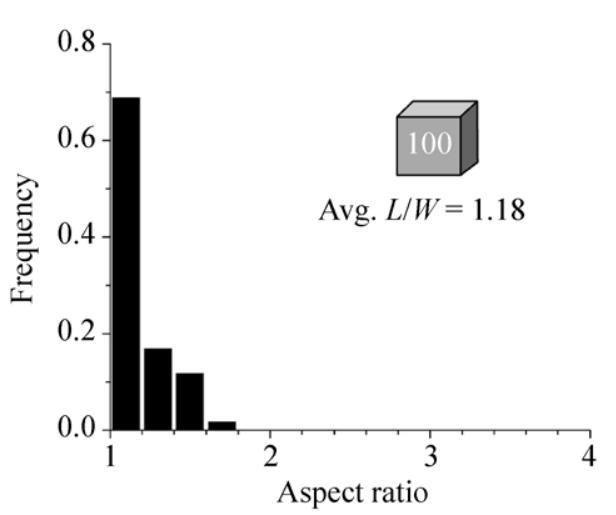

(b)

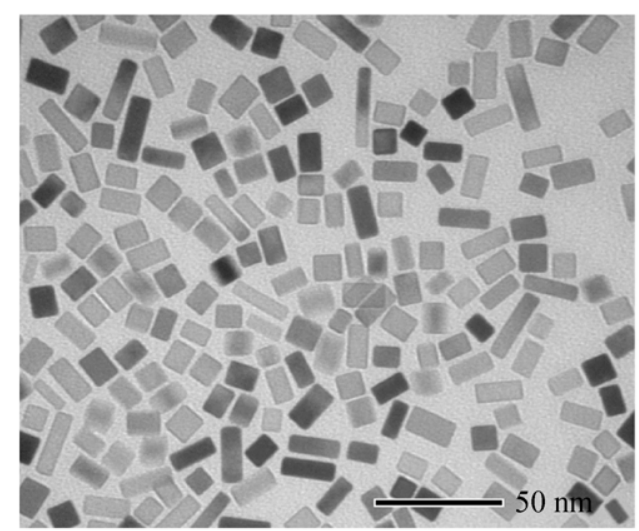

(c)

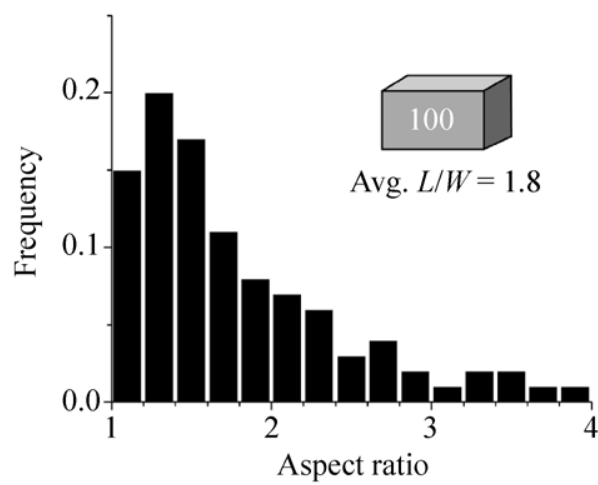

(d)

Figure 1 TEM images and aspect ratio (length/width, $L / W$ ) distributions of Pd nanobars synthesized at ((a), (b)) $80{ }^{\circ} \mathrm{C}$ and ((c), (d)) $100{ }^{\circ} \mathrm{C}$ 
in the Electronic Supplementary Material (ESM)). The same synthesis but at a higher temperature facilitated anisotropic growth and thus yielded Pd nanobars with higher aspect ratios. As shown in Figs. 1(c) and 1(d), the Pd nanobars synthesized at $100{ }^{\circ} \mathrm{C}$ exhibited an average aspect ratio of 1.8 , while the width remained at $8.3 \mathrm{~nm}$ (see Figs. S-1(c) and S-1(d) in the ESM).

We investigated the morphological evolution of $\mathrm{Pd}$ nanobars by taking samples at various reaction stages and then analyzing them by TEM. Figures 2(a)-2(d) show TEM images of samples obtained at $20 \mathrm{~s}, 40 \mathrm{~s}$, $1 \mathrm{~min}$, and $5 \mathrm{~min}$ into a reaction that was conducted at $80{ }^{\circ} \mathrm{C}$. At the very early stages of the reaction (Figs. 2(a) and $2(b))$, we observed the formation of a number of small particles with sizes less than $3 \mathrm{~nm}$ in addition to some large particles due to the coalescence of small particles. In high-resolution TEM (HRTEM) images of the large particles (Figs. 3(a) and $3(b))$, bottlenecks between the adjacent, smaller particles can be clearly seen, indicating that the coalescence events occurred between initially formed, small Pd particles. As the reaction proceeded to $t=1$ min, the number of small particles decreased and the large particles evolved into a bar-like morphology (Fig. 2(c)). In the following $4 \mathrm{~min}$, essentially all the small particles disappeared and the remaining $\mathrm{Pd}$ nanobars exhibited sharper edges and corners, as well as slightly increased sizes (Fig. 2(d)).

Growth via particle coalescence was also observed in the reaction conducted at $100{ }^{\circ} \mathrm{C}$ (Figs. 2(e)-2(h)). In this case, however, many of the coalesced particles exhibited an elongated structure as shown in
Fig. 2(f). HRTEM images of a coalesced particle obtained at $20 \mathrm{~s}$ into the reaction revealed that it contained twin defects and stacking faults in its crystal structure (Figs. 3(c) and 3(d)). Such defects are

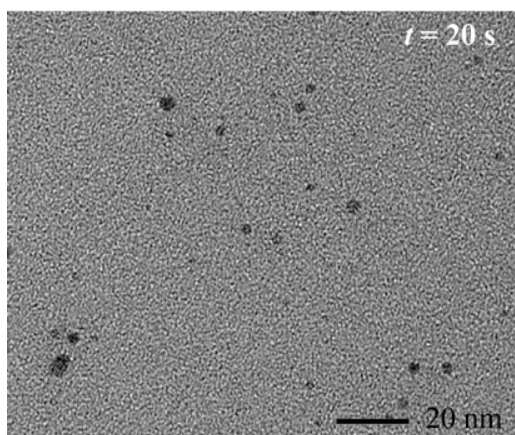

(a)

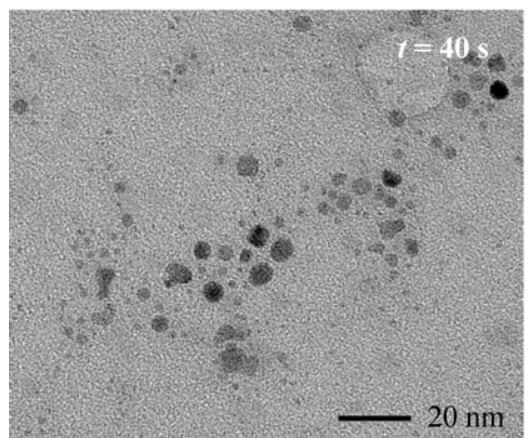

(b)

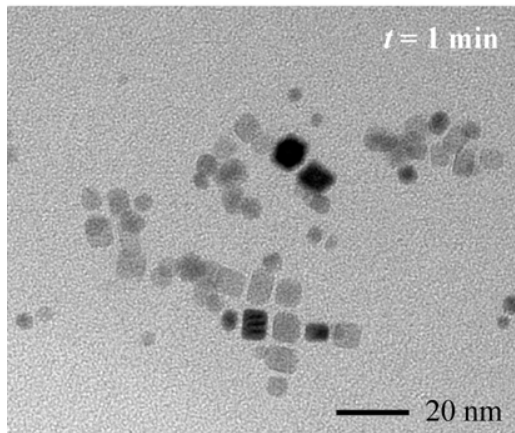

(c)

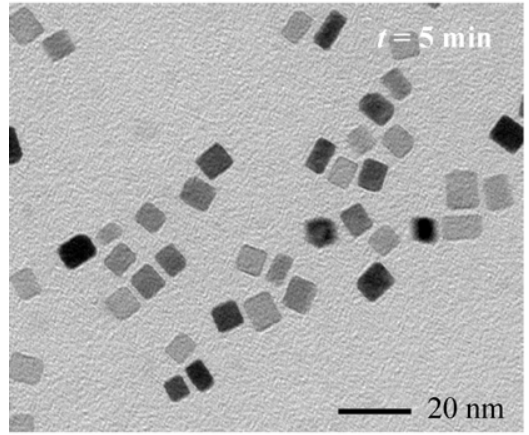

(d)

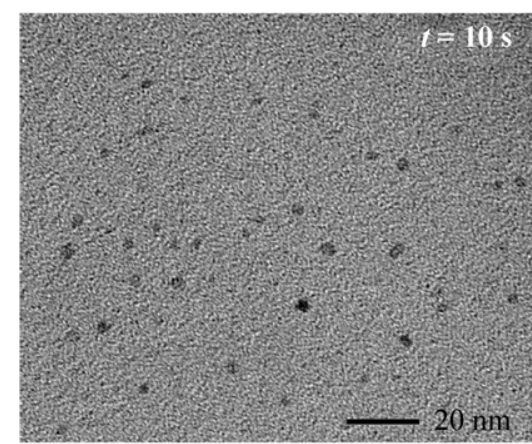

(e)

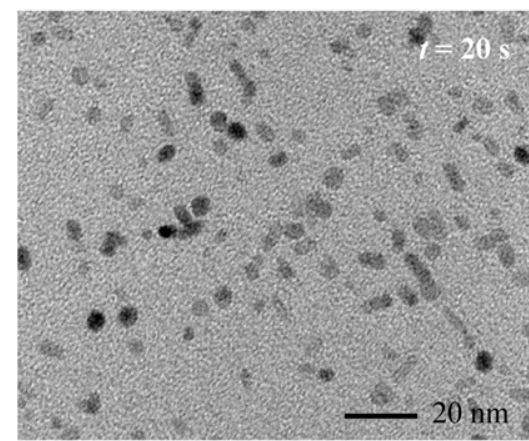

(f)

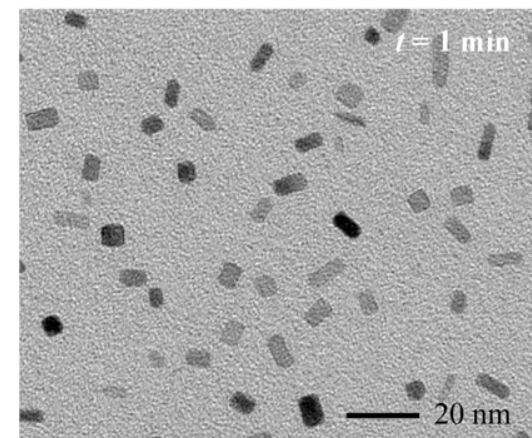

(g)

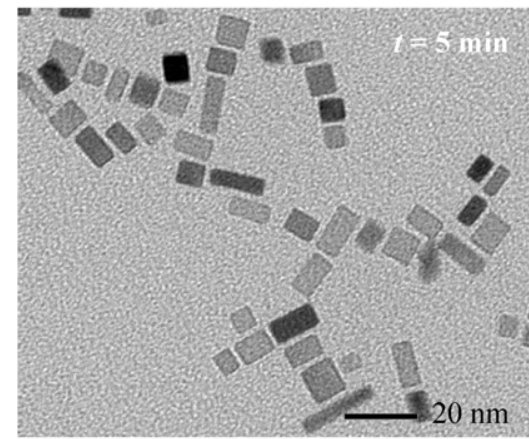

(h)
Figure 2 TEM images showing the morphological evolution of Pd nanobars synthesized at $80{ }^{\circ} \mathrm{C}((\mathrm{a})-(\mathrm{d}))$ and $100{ }^{\circ} \mathrm{C}((\mathrm{e})-(\mathrm{h}))$ 


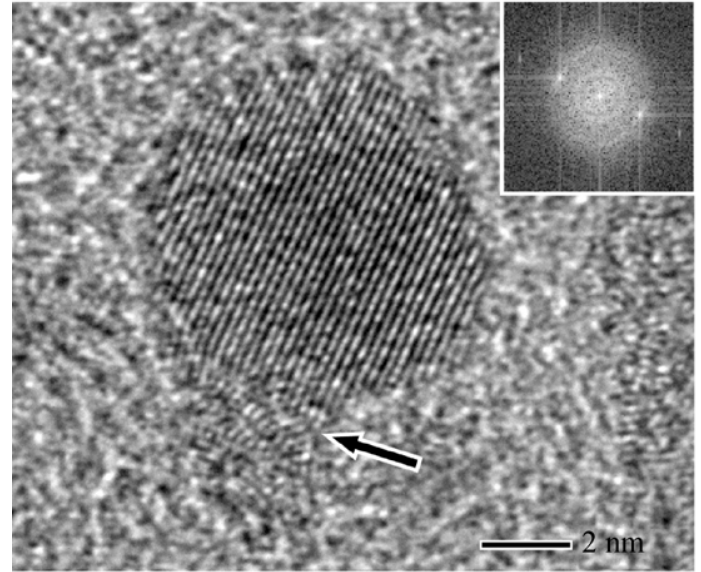

(a)

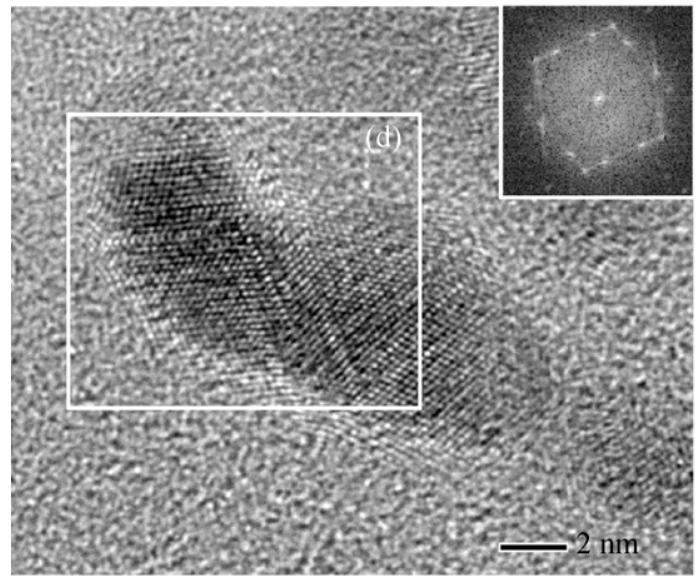

(c)

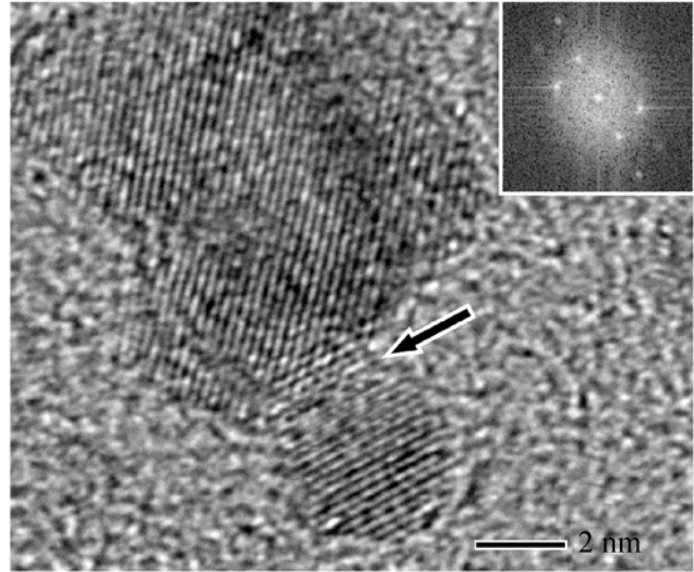

(b)

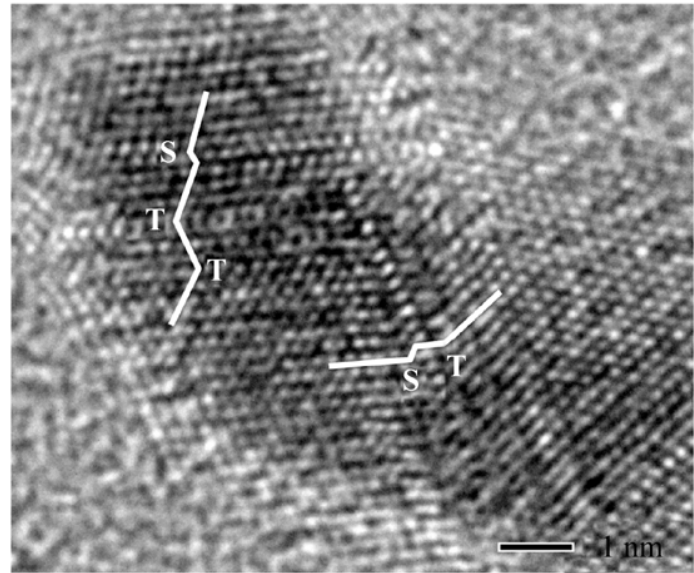

(d)

Figure 3 (a) and (b) HRTEM images of coalesced Pd particles obtained at $40 \mathrm{~s}$ into the reaction that was conducted at $80{ }^{\circ} \mathrm{C}$. (c) HRTEM image of a coalesced Pd particle obtained at $20 \mathrm{~s}$ into the reaction that was conducted at $100{ }^{\circ} \mathrm{C}$. (d) Magnified image of the boxed region in (c). $\mathrm{T}$ and $\mathrm{S}$ denote twin defect and stacking fault, respectively. In (a)-(c), the insets show the corresponding Fourier transform (FT) patterns

common features of an oriented attachment growth mechanism and have also been observed in the synthesis of nanorods and nanowires made of metals or metal sulfides $[22,23]$. In the previous studies, a mechanism based on the anisotropic growth of initially formed nanocubes via localized oxidative etching has been used to explain the formation of $\mathrm{Pd}$ nanobars with aspect ratios larger than unity $[25,26]$. As demonstrated in the current study, however, this type of $\mathrm{Pd}$ nanostructure seems to be formed via particle coalescence in a one-dimensional fashion even though the exact pathway for such growth is yet to be resolved.

Particle coalescence can be attributed to the fact that small particles have a higher chemical potential than large particles due to a larger surface-to-volume ratio, which can give rise to an abrupt increase in particle size. In the synthesis of Pd nanobars, particle size rapidly increased at the very early stages of the reaction (Fig. 4), due to the involvement of coalescence in the growth process. After this stage, particle size increased gradually and continuously until it reached a saturation stage. Growth via atomic addition that occurs throughout the reaction should dominate the
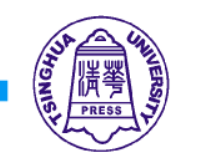

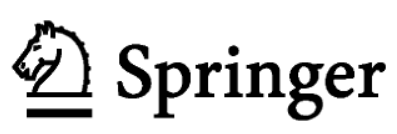




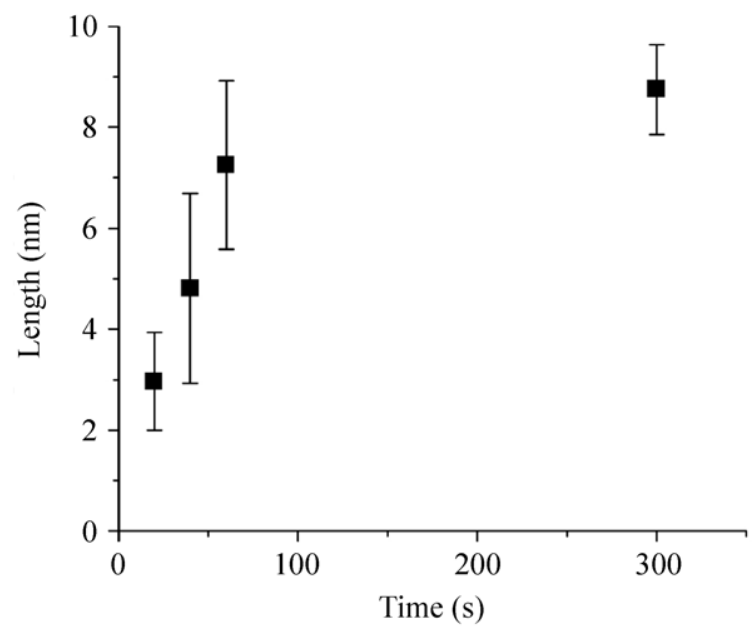

(a)

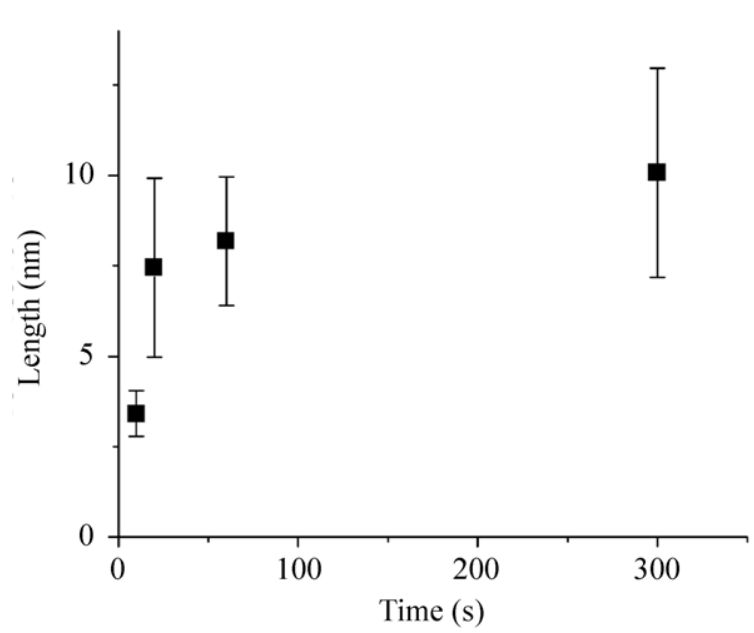

(b)

Figure 4 Particle length versus time for the reactions conducted at (a) $80{ }^{\circ} \mathrm{C}$ and (b) $100{ }^{\circ} \mathrm{C}$

continued growth at the later stages of the reaction. Although the initial, coalesced particles had irregular morphologies, all the final nanocrystals exhibited the shape of a nanobar, without inclusion of any bumps on their surfaces. These observations suggest that particle coalescence was accompanied by shape focusing via recrystallization of the attached particles, which has also been observed in the syntheses of $\mathrm{Pt}$ and $\mathrm{Pd}-\mathrm{Pt}$ alloy nanocrystals $[16,24]$. The recrystallization process involves the migration of atomic species on the two-dimensional surface of a nanocrystal, giving rise to reconstruction of the surface and thus shape. During this shape focusing process, bromide ions seem to play a significant role in promoting the formation of
$\{100\}$ facets by lowering their surface energy through the preferential chemisorption [25-27], and thus inducing evolution of the shape into a nanobar.

Metal nanocrystals with well-defined shapes such as cubes or octahedra have often been described by assuming the absence of surface defects on their main faces. In order to elucidate the detailed surface structure of the Pd nanobars, we performed a sub-Ångström resolution, HAADF-STEM analysis. Figure 5(a) shows an HAADF-STEM image of a nanobar with an aspect ratio of 1.08. The spacing between lattice fringes was $1.94 \AA$, which can be indexed as $\{200\}$ of Pd with a face-centered cubic (fcc) packing. The image displayed highly ordered, continuous fringes with the same orientation, demonstrating that the faces of the nanobar were enclosed by $\{100\}$ facets, albeit the corners were slightly truncated. The nanobar did not contain any twin defects or stacking faults in its crystal structure, indicating that it was a piece of single crystal. Interestingly, however, we observed the formation of several types of surface defects on the $\{100\}$ faces of the nanobar, including an adatom island, a vacancy pit, and steps as shown in Figs. 5(b)-5(e). Such defects are often observed in thin-film growth via atomic deposition from the gas phase, where the deposited atoms nucleate on a two-dimensional surface and various types of defects can form depending on the mobility of surface atoms [28]. Our results suggest that a two-dimensional nucleation and growth process was also involved in the surface evolution of the $\mathrm{Pd}$ nanobars via atomic addition from the solution phase.

We also examined the thermal behavior of the $\mathrm{Pd}$ nanobars by annealing them inside an electron microscope chamber at $500{ }^{\circ} \mathrm{C}$ for $1 \mathrm{~h}$. Note that the $\mathrm{Pd}$ sample was washed prior to the annealing experiments in an effort to remove the residual bromide ions. As shown in Fig. 6(a), the annealing process made the nanobar more severely truncated at the corners. This observation implies that the shape transformation process involved the migration of surface atoms from the corners to the main faces (Fig. 6(b)), resulting in the enlargement of $\{111\}$ facets and the shrinkage of $\{100\}$ facets. For an fcc metal, the surface energies of low-index facets increase in the 


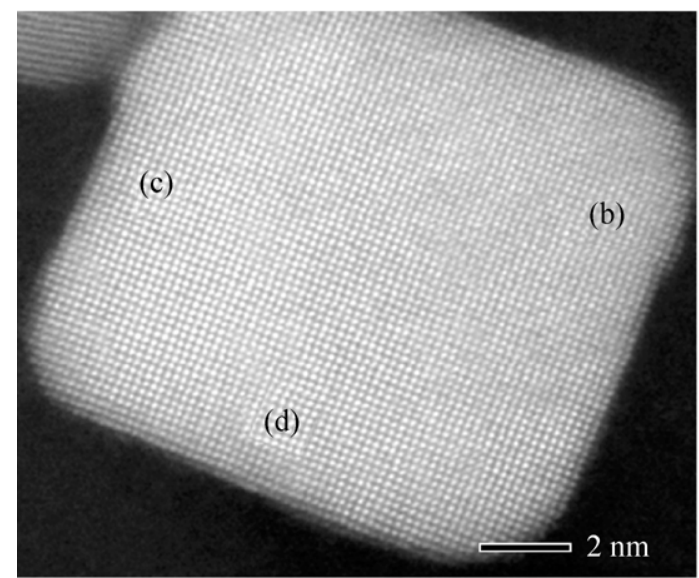

(a)

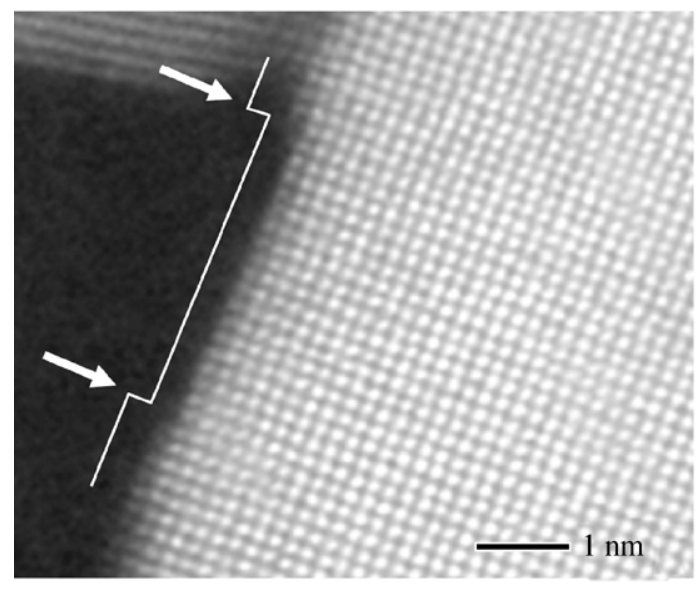

(c)

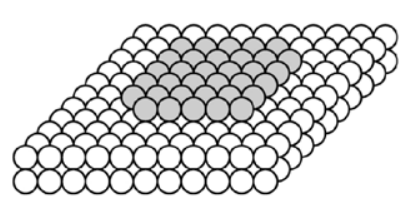

Island

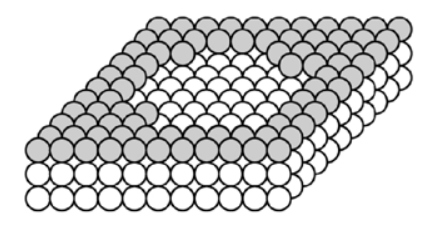

Vacancy pit

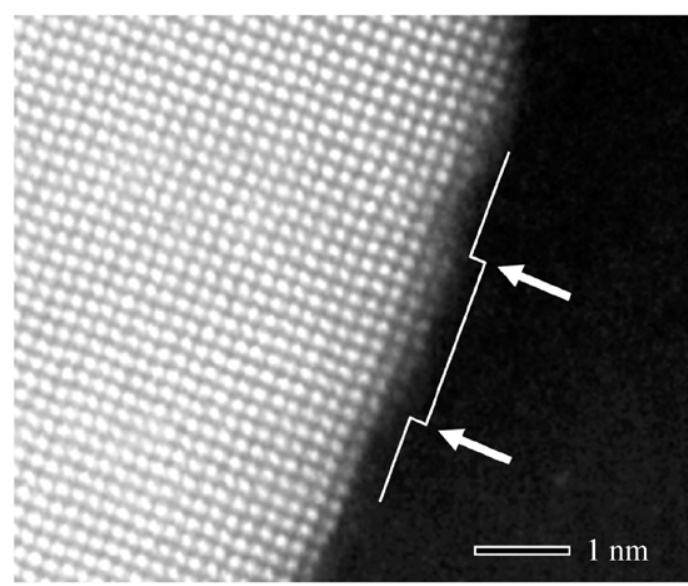

(b)

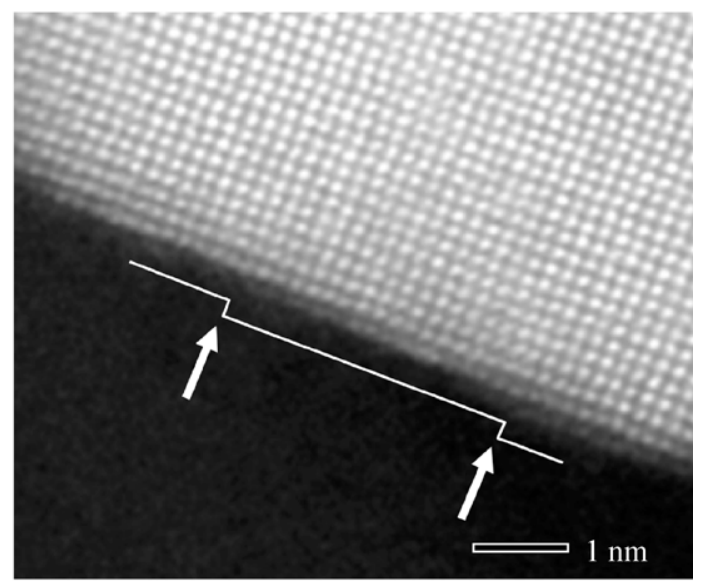

(d)

(e)

Figure 5 (a) HAADF-STEM image of an as-prepared, single Pd nanobar. (b)-(d) Magnified images of the selected regions in (a). Individual Pd atoms are resolved as white spots. (e) Schematic models of the $\{100\}$ surfaces containing an island (left), a vacancy pit (middle), and a step (right)

order of $\gamma\{111\}<\gamma\{100\}<\gamma\{110\}$. Considering that the annealing process is mainly governed by thermodynamics, the enlargement of $\{111\}$ facets with the lowest surface energy should be a major driving force for such transformation. It is worth pointing out that the nanobar was defect free on its $\{100\}$ faces (Figs. 6(c) and 6(d)), indicating that surface reconstruction also occurred during the annealing process. This can be attributed to the higher energy of atoms on the edges of the defects due to their relatively low coordination numbers.

In summary, we have investigated the growth mechanism involved in the aqueous synthesis of $\mathrm{Pd}$ nanobars. We have shown that growth of the $\mathrm{Pd}$ nanobars was dominated by particle coalescence at the very early stages of the synthesis, which was 


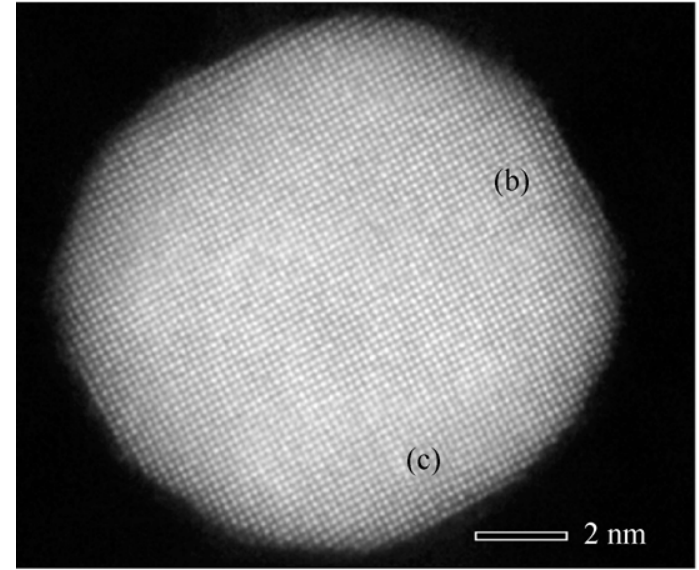

(a)

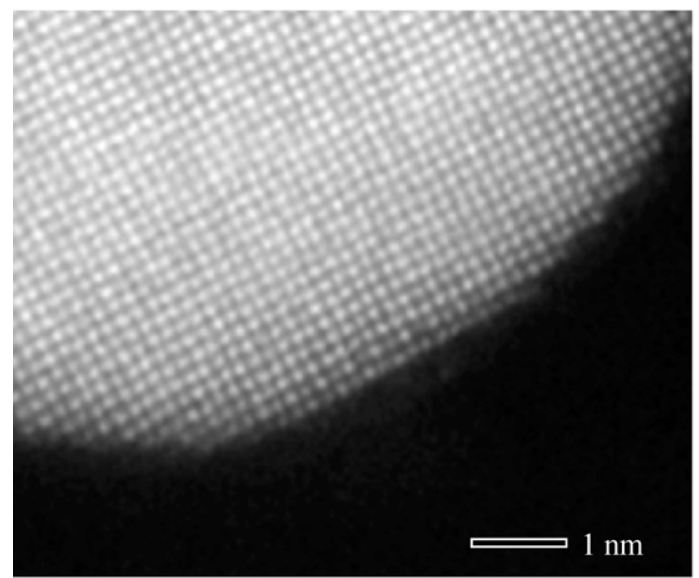

(c)

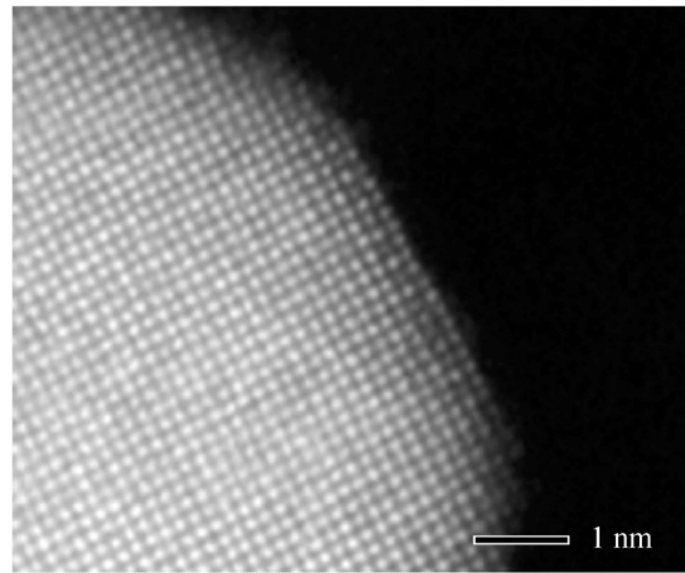

(b)

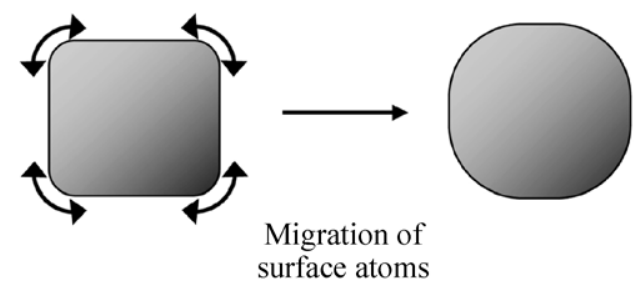

(d)

Figure 6 (a) HAADF-STEM image taken from a Pd nanobar supported on a carbon film after in situ annealing inside the electron microscope chamber at $500{ }^{\circ} \mathrm{C}$ for $1 \mathrm{~h}$. ((b), (c)) Magnified images of the selected regions in (a). Individual Pd atoms are resolved as white spots. (d) Schematic illustration of the migration of surface atoms during the annealing process

followed by shape focusing via recrystallization and further size growth via atomic addition. The as-synthesized Pd nanobars exhibited several types of defects such as an adatom island, a vacancy pit, and steps on their exposed $\{100\}$ surfaces. This work not only greatly advances our understanding of the growth mechanism of $\mathrm{Pd}$ nanocrystals, but also provides new insights into their surface structure at an atomic scale.

\section{Experimental}

Synthesis of Pd nanobars. In a typical synthesis of $\mathrm{Pd}$ nanobars, $11 \mathrm{~mL}$ of an aqueous solution containing poly(vinyl pyrrolidone) (PVP, $M_{\mathrm{W}} \approx 55000,105 \mathrm{mg}$, Aldrich), L-ascorbic acid (60 mg, Aldrich), $\mathrm{KBr}$ (300 mg, Fisher), and $\mathrm{Na}_{2} \mathrm{PdCl}_{4}$ (57 mg, Aldrich) was heated at a temperature of either 80 or $100{ }^{\circ} \mathrm{C}$ in air under magnetic stirring for $3 \mathrm{~h}$ and then cooled down to room temperature.

Microscopy characterization. In the preparation of samples for TEM and HRTEM studies, aliquots of the reaction solution were rapidly cooled by adding precooled acetone. The resulting solutions were dropped directly onto carbon-coated copper grids without centrifugation and dried inside the vacuum chamber of a freeze-drier. After drying, the grids were washed 
with ethanol to remove the remaining PVP, and dried again. TEM and HRTEM images were captured using a Phillips 420 microscope operated at $120 \mathrm{kV}$ and a JEOL 2100F microscope operated at $200 \mathrm{kV}$, respectively. Sub-Ångström resolution, HAADF-STEM images were acquired on a JEOL 2200FS STEM/TEM instrument equipped with a CEOS $\mathrm{GmbH}$ probe corrector, providing a nominal image resolution of $0.07 \mathrm{~nm}$. The sample heating was accomplished by utilizing the Protochips Aduro ${ }^{\mathrm{TM}}$ heating technology.

\section{Acknowledgements}

This work was supported in part by the Natural Science Foundation (No. DMR-0804088) and startup funds from Washington University in St. Louis. P. H. C. C. was also partially supported by the Fulbright Program and the Brazilian Ministry of Education (CAPES). Part of the work was performed at the Nano Research Facility (NRF), a member of the National Nanotechnology Infrastructure Network (NNIN), which is supported by the National Science Foundation (No. ECS-0335765). It was also supported by startup funds from the University of Missouri-St. Louis. The STEM images were acquired at the Oak Ridge National Laboratory's High Temperature Materials Laboratory sponsored by the U.S. Department of Energy, Office of Energy Efficiency and Renewable Energy, Vehicle Technologies Program.

Electronic Supplementary Material: Width and length distributions of $\mathrm{Pd}$ nanobars synthesized at different temperatures are available in the online version of this article at http://dx.doi.org/10.1007/s12274-010-1021-5 and are accessible free of charge.

Open Access: This article is distributed under the terms of the Creative Commons Attribution Noncommercial License which permits any noncommercial use, distribution, and reproduction in any medium, provided the original author(s) and source are credited.

\section{References}

[1] Skrabalak, S. E.; Chen, J.; Sun, Y.; Lu, X.; Au, L.; Cobley,
C. M.; Xia, Y. Gold nanocages: Synthesis, properties, and applications. Acc. Chem. Res. 2008, 41, 1587-1595.

[2] Xia, Y.; Xiong, Y.; Lim, B.; Skrabalak, S. E. Shapecontrolled synthesis of metal nanocrystals: Simple chemistry meets complex physics? Angew. Chem. Int. Ed. 2009, 48, 60-103.

[3] Peng, Z.; Yang, H. Designer platinum nanoparticles: Control of shape, composition in alloy, nanostructure and electrocatalytic property. Nano Today 2009, 4, 143-164.

[4] Tian, N.; Zhou, Z. -Y.; Sun, S. -G.; Ding, Y.; Wang, Z. L. Synthesis of tetrahexahedral platinum nanocrystals with high-index facets and high electro-oxidation activity. Science 2007, 316, 732-735.

[5] Bratlie, K. M.; Lee, H.; Komvopoulos, K.; Yang, P.; Somorjai, G. A. Platinum nanoparticle shape effects on benzene hydrogenation selectivity. Nano Lett. 2007, 7, 3097-3101.

[6] Lim, B.; Lu, X.; Jiang, M.; Camargo, P. H. C.; Cho, E. C.; Lee, E. P.; Xia, Y. Facile synthesis of highly faceted multioctahedral Pt nanocrystals through controlled overgrowth. Nano Lett. 2008, 8, 4043-4047.

[7] Wang, C.; Daimon, H.; Onodera, T.; Koda, T.; Sun, S. A general approach to the size- and shape-controlled synthesis of platinum nanoparticles and their catalytic reduction of oxygen. Angew. Chem. Int. Ed. 2008, 47, 3588-3591.

[8] Lim, B.; Jiang, M.; Camargo, P. H. C.; Cho, E. C.; Tao, J.; Lu, X.; Zhu, Y.; Xia, Y. Pd-Pt bimetallic nanodendrites with high activity for oxygen reduction. Science 2009, 324, 1302-1305.

[9] Habas, S. E.; Lee, H.; Radmilovic, V.; Somorjai, G. A.; Yang, P. Shaping binary metal nanocrystals through epitaxial seeded growth. Nat. Mater. 2007, 6, 692-697.

[10] LaMer, V. K.; Dinegar, R. H. Theory, production and mechanism of formation of monodispersed hydrosols. J. Am. Chem. Soc. 1950, 72, 4847-4854.

[11] Peng, X.; Wickham, J.; Alivisatos, A. P. Kinetics of II-VI and III-V colloidal semiconductor nanocrystal growth: "Focusing" of size distributions. J. Am. Chem. Soc. 1998, 120, 5343-5344.

[12] Park, J.; Joo, J.; Kwon, S. G.; Jang, Y.; Hyeon, T. Synthesis of monodisperse spherical nanocrystals. Angew. Chem. Int. Ed. 2007, 46, 4630-4660.

[13] Anwar, J.; Boateng, P. K. Computer simulation of crystallization from solution. J. Am. Chem. Soc. 1998, 120, 9600-9604.

[14] Niederberger, M.; Colfen, H. Oriented attachment and mesocrystals: Non-classical crystallization mechanisms based on nanoparticle assembly. Phys. Chem. Chem. Phys. 2006, $8,3271-3287$. 
[15] Watzky, M. A.; Finney, E. E.; Finke, R. G. Transition-metal nanocluster size vs. formation time and the catalytically effective nucleus number: A mechanism-based treatment. J. Am. Chem. Soc. 2008, 130, 11959-11969.

[16] Lim, B.; Wang, J.; Camargo, P. H. C.; Cobley, C. M.; Kim, M. J.; Xia, Y. Twin-induced growth of palladium-platinum alloy nanocrystals. Angew. Chem. Int. Ed. 2009, 48, 6304-6308.

[17] Bisson, L.; Boissiere, C.; Nicole, L.; Grosso, D.; Jolivet, J. P.; Thomazeau, C.; Uzio, D.; Berhault, G.; Sanchez, C. Formation of palladium nanostructures in a seed-mediated synthesis through an oriented-attachment-directed aggregation. Chem. Mater. 2009, 21, 2668-2678.

[18] Banfield, J. F.; Welch, S. A.; Zhang, H.; Ebert, T. T.; Penn, R. L. Aggregation-based crystal growth and microstructure development in natural iron oxyhydroxide biomineralization products. Science 2000, 289, 751-754.

[19] Pacholski, C.; Kornowski, A.; Weller, H. Self-assembly of $\mathrm{ZnO}$ : From nanodots to nanorods. Angew. Chem. Int. Ed. 2002, 41, 1188-1191.

[20] Tang, Z.; Kotov, N. A.; Giersig, M. Spontaneous organization of single CdTe nanoparticles into luminescent nanowires. Science 2002, 297, 237-240.

[21] Zhang, Z.; Tang, Z.; Kotov, N. A.; Glotzer, S. C. Simulations and analysis of self-assembly of CdTe nanoparticles into wires and sheets. Nano Lett. 2007, 7, 1670-1675.

[22] Yu, J. H.; Joo, J.; Park, H. M.; Baik, S. -I.; Kim, Y. W.; Kim, S. C.; Hyeon, T. Synthesis of quantum-sized cubic ZnS nanorods by the oriented attachment mechanism. J. Am. Chem. Soc. 2005, 127, 5662-5670.

[23] Halder, A.; Ravishankar, N. Ultrafine single-crystalline gold nanowire arrays by oriented attachment. Adv. Mater. 2007, 19, 1854-1858.

[24] Zheng, H.; Smith, R. K.; Jun, Y. -W.; Kisielowski, C.; Dahmen, U.; Alivisatos, A. P. Observation of single colloidal platinum nanocrystal growth trajectories. Science 2009, 324, 1309-1312.

[25] Lim, B.; Jiang, M.; Tao, J.; Camargo, P. H. C.; Zhu, Y.; Xia, Y. Shape-controlled synthesis of $\mathrm{Pd}$ nanocrystals in aqueous solutions. Adv. Funct. Mater. 2009, 19, 189-200.

[26] Xiong, Y.; Cai, H.; Wiley, B. J.; Wang, J.; Kim, M. J.; Xia, Y. Synthesis and mechanistic study of palladium nanobars and nanorods. J. Am. Chem. Soc. 2007, 129, 3665-3675.

[27] Niu, W.; Li, Z. -Y.; Shi, L.; Liu, X.; Li, H.; Han, S.; Chen, J.; $\mathrm{Xu}, \mathrm{G}$. Seed-mediated growth of nearly monodisperse palladium nanocubes with controllable sizes. Cryst. Growth Des. 2008, 8, 4440-4444.

[28] Zhang, Z.; Lagally, M. G. Atomistic processes in the early stages of thin-film growth. Science 1997, 276, 377-383. 